was admitted at 26 weeks gestation under mental health legislation due to cognitive impairment and self-neglect.

Method She was commenced on darunavir/ritonavir $600 \mathrm{mg}$ bd, truvada and raltegravir but three weeks later, at 29 weeks gestation, she developed rapidly progressive hepatic transaminitis. Abdominal ultrasound scan was normal and tests for viral hepatitis negative. Pre-eclampsia was excluded, leaving three working diagnoses: drug-induced hepatitis, obstetric cholestasis or acute fatty liver of pregnancy. ARVs were stopped but transaminases continued to rise (ALT 614 and AST $716 \mathrm{U} / \mathrm{L}$ ). Clotting screen and platelet count remained normal but the patient began to complain of epigastric pain. HIV viral load had risen to 241 copies $/ \mathrm{ml}$. In view of deteriorating maternal health and the increasing risk of MTCT (HIV viral load expected to rise), the baby was delivered at 31 weeks' gestation by semi-elective caesarean after a course of antenatal steroids. The baby received antiviral prophylaxis in the form of abacavir, lamivudine and zidovudine; HIV RNA was undetectable at three months (MTCT extremely unlikely). Nine days after delivery the patient's LFTs normalised. Conclusion Darunavir-induced hepatitis typically presents with increased AST and ALT. In this case, LFTs only started to improve following delivery of the baby, suggesting a pregnancy related cause.

\section{P47 HIV SEROCONVERSION IN PREGNANCY RUNS AN INCREASED RISK OF MOTHER TO CHILD TRANSMISSION (MTCT)}

Rebecca Acquah*, Fiona Fargie, Andrew Winter. Sandyford Sexual Health Service, NHS Greater Glasgow and Clyde, Glasgow, UK

\subsection{6/sextrans-2015-052126.91}

Background We present the case of a couple who attended our sexual health service - him with a Severe Primary Herpes episode and other indicators of immune compromise and her in her $41^{\text {st }}$ week of pregnancy. Their last sexual contact was nine days previously. Urgent HIV testing was undertaken using a fourth generation test with the male partners' test being positive and the female partners' test being negative. Viral load testing was requested with a result anticipated in $24 \mathrm{~h}$.

Method During the night his partner went into labour. We calculated the risk of MTCT in this unique situation as being approximately $1: 4000$ and advised the patient that this could be decreased to 1:10 000 with Nevirapine, Zidovudine and a delivery by caesarean section. The baby received triple drug antiviral therapy until a negative viral load was confirmed approximately $6 \mathrm{~h}$ after delivery. Due to the risk of seroconversion the mother decided not to breastfeed even with antiretroviral cover, although sterilisation of expressed breast milk was discussed. Management of serodiscordant couples during pregnancy with ongoing risk of transmission is not discussed in the BHIVA guidelines and there is little evidence/guidance to base decisions around breastfeeding and retesting on.

Conclusion We wonder if we had been able to get a viral load on the female sample more quickly, would it have prevented caesarean section or would concerns around risk of acquisition from the genital tract during vaginal delivery (should she be in the 'eclipse' phase of HIV) have still made us advise an operative delivery.
P48

MYCOBACTERIAL SPINDLE CELL PSEUDOTUMOUR IN A PATIENT WITH HIV

Eoin Walker*, Emma McCarty, Claire Donnelly, Carol Emerson, Say Quah. Royal Victoria Hospital, Belfast, UK

\subsection{6/sextrans-2015-052126.92}

Background/introduction Mycobacterial spindle cell pseudotumour is a rare, benign lesion caused by local proliferation of histiocytes in response to mycobacterial infection. It most commonly occurs with mycobacterium avium intracellulare. Most cases affect lymph nodes, skin and brain. We present a case occurring in the lung of a patient with HIV.

Methods A 38 year old Caucasian gentleman was admitted with 1 year history of weight loss, cough and diarrhoea. As a result of declining health and recent HIV diagnosis, he had returned to UK after living 8 years in Thailand. He had commenced anti-TB drugs 6 weeks previously; however no details were available regarding previous investigations. He was profoundly immunosuppressed, with CD4 count $<10$ copies $/ \mathrm{mm}^{3}$. CT chest showed widespread cavitating lesions throughout both lung fields. Cultures from sputum and bronchial washings grew mycobacterium avium intracellulare and clarithromycin was added. Antiretroviral treatment was started 2 weeks later. Biopsies from bone marrow and bowel showed no evidence of granuloma or malignancy. He suffered frequent episodes of hypercalcaemia. As a result of this, and lack of radiological response to mycobacterial treatment and ARV, CT guided lung biopsy was carried out. This showed mycobacterial spindle cell pseudotumour. Clinically he continued to improve, with immune recovery. Anti-mycobacterial treatment was to continue for 12 months.

Discussion/conclusion Mycobacterial spindle cell pseudotumour is a rare complication of mycobacterial infection. The majority of patients are immunocompromised, including those with advanced HIV. It may share some histological features with Kaposi Sarcoma, therefore correct identification is essential. Treatment depends on the mycobacterial species identified.

\section{P49 TOXIC CARDIOMYOPATHY IN A STABLE HIV PATIENT WITH A HISTORY OF AMPHETAMINE MISUSE-A CASE REPORT}

Durba Raha*, Imali Fernando. Chalmers Sexual Health Centre, Edinburgh, USA

\subsection{6/sextrans-2015-052126.93}

Background/introduction Amphetamine (AM) use is associated with HIV infection among MSM. There are various toxic effects of AM, cardiotoxicity being one of them.

Aim(s)/objectives To present a case of report of cardiomyopathy secondary to AM misuse in a patient with well-controlled HIV.

Case report A 51 year old HIV positive MSM was admitted to hospital with dyspnoea, orthopnoea and decreased exercise tolerance. He was HIV positive since 1990 and this is stable on ARVs. CD4 count pre-admission was 514 with undetectable viral load. He used 25-30 grams of AM per week over a period of 20 years and had multiple casual unprotected MSM partners. On admission, the patient was tachycardic and hypoxic. Chest $\mathrm{X}$-Ray on admission showed cardiomegaly and bi-basal opacification. Echocardiogram demonstrated severe left and right 
ventricular dysfunction, at a level requiring cardiac transplant. ECG showed prolonged QT interval. The patient was diagnosed with toxic dilated cardiomyopathy secondary to long term AM abuse. UK guidelines for Heart transplantation in adults deem chronic viral infection and ongoing substance misuse as relative contraindications to transplant. He was consequently commenced on medication for cardiac failure and received benzodiazepine as inpatient for managing withdrawal symptoms. On discharge, psychiatry follow-up was organised for support to help reduction of AM. At follow up, the patient reported reduced AM use by quarter, but felt he could never abstain.

Discussion/conclusion AM related cardiac fatalities are caused by acute myocardial necrosis, ventricular rupture, cardiomyopathy or arrhythmia. Evidence is mostly derived from casereports. Patients using AM should be fully counselled regarding possible toxic effects.

\section{\begin{tabular}{|l|l}
\hline P50 NON-ISCHAEMIC DILATED CARDIOMYOPATHY IN HIV \\
\hline
\end{tabular} POSITIVE PATIENTS; A CASE SERIES}

Larissa Mulka*, Catherine Kirby, Duncan Churchill. Lawson Unit, Royal Sussex County Hospital, Brighton, UK

\subsection{6/sextrans-2015-052126.94}

Background HIV is a well-known cause of dilated cardiomyopathy, with an annual incidence of 15.9 per 1000 asymptomatic HIV patients in the pre-HAART era. Despite reduced incidence with HAART, it remains an important cause of cardiac morbidity in people with HIV though its direct association to the virus is unclear.

Methods Retrospective case review.

Results Four patients with dilated cardiomyopathy were identified out of 4739 attending between 2002-2014. Mean age was 49 years (range 38-62), all were male. Two presented as admissions with cardiac failure; two were diagnosed on routine investigation for exertional dyspnoea. All clinically improved with medical management; the three cases under long term follow up (6-10 years) showed improvement in ejection fraction (EF), though one died 10 years post diagnosis of presumed suddencardiac death.

Discussion This small case series highlights the positive outcomes with medical management of dilated cardiomyopathy in HIV. The direct role of HIV remains unclear; these cases reinforce the importance of regular screening for recreational drug use and consideration of their potential cardiotoxicity, and awareness of other aetiological factors.

\section{Category: Electronic patient records and use of information technology}

\section{P51 THE UTILITY OF PERSONALISED SHORT MESSAGE SERVICE (SMS) TEXTS TO REMIND PATIENTS AT HIGHER RISK OF STIS AND HIV TO RE-ATTEND FOR TESTING}

Farai Nyatsanza*, Siobhan Murphy, John McSorley, Gary Brook. Central Middlesex Hospital, London, UK

10.1136/sextrans-2015-052126.95
Background Patients at increased risk of STI/HIV acquisition are advised to re-attend for re-testing. A previous study showed that 'generic' text reminders did not improve re-attendance.

Aim To assess if a personalised text message would increase reattendance rates of at risk patients who require repeat STI testing. Methods At-risk patients were sent a text reminder to re-attend for re-testing 6 weeks after their initial visit. They were considered to be 'at risk' if they had an acute STI or had attended for emergency contraception at the initial visit, or were MSM. Re-attendance rates were measured for September to December 2012 (control group who received a generic text message advising re-attendance) and February to May 2014 (personalised message group who received a text message containing their first name and several different ways to contact the clinic). Re-attendance was counted within four months of the end of the initial episode of care.

Results The re-attendance rate was significantly higher for the personalised message group $(144 / 266(54 \%)$ than the control group: $(90 / 273(33 \%)(\mathrm{P}=0.0001)$ and was also significantly higher in the personalised message group than the control group in patients with the following risks: recent chlamydia (61/123 $(50 \%)$ vs $(43 / 121(36 \%)(\mathrm{P}=0.03)$, recent gonorrhoea $(42 / 64$ $(66 \%)$ vs $(4 / 21(19 \%)(\mathrm{P}=0.0003)$ and MSM $(25 / 45(56 \%)$ vs $(3 / 18(16 \%) \quad(\mathrm{P}=0.006)$. New STI rates in the re-attending 'personalised message' group and controls were 26/144(18\%) and 13/90 (14\%) (n.s) respectively.

Conclusion Sending a personalised text message as a reminder for re-testing increases re-attendance rates in patients who are at higher risk of STIs.

\section{P52 KEEPING "APP" TO DATE: USING GEOLOCATION APPS TO SIGNPOST TO LOCAL SEXUAL HEALTH SERVICES}

Richard West, Elizabeth Okecha*, Kimberley Forbes. Sexual Health Hounslow, London, UK

\subsection{6/sextrans-2015-052126.96}

Background/introduction To encourage HIV testing amongst men who have sex with men (MSM) during "National HIV testing week" (NHTW) point of care testing (POCT) was offered at community and hospital-based sexual health services (SHS). Users of the social networking application "Grindr" within 5 miles of our clinics received a link to our website, which was upgraded to include a video demonstrating HIV POCT. Traditional health promotion poster campaign was also employed.

Aim(s)/objectives To review advertising strategies used and clients who requested POCT during NHTW.

Methods Activity data was obtained from the software company and electronic records of those attending for POCT were reviewed.

Results 43 asymptomatic attendees requested POCT testing, 35 male and 8 female. 21 males identified as MSM (60\%), $15(71 \%)$ disclosed that they had attended as a result of the "Grindr" advertisement. The average MSM number of daily visits to the website increased from 250 to 600/ day, highest at weekends the majority via "Grindr". POCT video was viewed 126 times during testing week. $30(70 \%)$ patients accepted a sexual health screen, 3 asymptomatic infections were diagnosed. No HIV diagnoses were made.

Discussion/conclusion Social networking proved popular amongst MSM. No HIV diagnoses were made however screening increased HIV testing and identified sexual infections in 